Bioinformation

www.bioinformation.net

\title{
Evolutionary trace analysis at the ligand binding site of laccase
}

\author{
Saharuddin Bin Mohamad ${ }^{1, *}$, Ai Ling Ong ${ }^{2}$ and Adiratna Mat Ripen ${ }^{3}$ \\ ${ }^{1}$ Institute of Biological Sciences, University of Malaya, 50603 Kuala Lumpur, Malaysia; ${ }^{2}$ Multimedia University, Jalan Ayer Keroh \\ Lama, 75450 Melaka, Malaysia; ${ }^{3}$ Institute for Medical Research, Jalan Pahang, 50588 Kuala Lumpur, Malaysia; \\ Saharuddin Bin Mohamad* - E-mail: saharuddin@um.edu.my; * Corresponding author \\ received April 08, 2008; revised April 29, 2008; accepted May 07, 2008; published June 18, 2008
}

\begin{abstract}
:
Laccase belongs to the family of blue multi-copper oxidases and are capable of oxidizing a wide range of aromatic compounds. Laccases have industrial applications in paper pulping or bleaching and hydrocarbon bioremediation as a biocatalyst. We describe the design of a laccase with broader substrate spectrum in bioremediation. The application of evolutionary trace (ET) analysis of laccase at the ligand binding site for optimal design of the enzyme is described. In this attempt, class specific sites from ET analysis were mapped onto known crystal structure of laccase. The analysis revealed $162 \mathrm{PHE}$ as a critical residue in structure function relationship studies.
\end{abstract}

Keywords: evolutionary trace (ET) analysis; laccase; ligand binding site; enzyme bioremediation

\section{Background:}

White rot fungus such as Trametes versicolor is a good candidate for biodegradation of aromatic compound in soil. Their oxidative capacity using enzymatic methods for the transformation of aromatic compounds occur at an extra-cellular environment and hence do not require internalization prior to degradation. Among the oxidative enzymes, laccase from Trametes versicolor has been reported to be involved in degradation. Laccases (E.C. 1.10.3.2) are members of the ubiquitous blue multicopper oxidase family. They catalyze the oxidation of a range of organic substrates such as phenols, aromatic amines and non-phenolic compounds by reducing molecular oxygen to water [1].

Most of these enzymes are extra cellular in nature. In some species, they occur as isozymes in both extra- and intracellular environments. Laccases are widely distributed ranging from plants, insects, fungi to bacteria [2]. Their role in synthesis and/or degradation of the biopolymer lignin is well known [3]. However, their physiological function is still under intense investigation for potential improvement in biotransformation reaction kinetics. Laccases are exploited in biotechnology applications and soil/water bioremediation $[4,5]$.

The evolutionary trace (ET) analysis is developed by Olivier Lichtarge in 1996 [6]. This method relies on both sequence and structural information to analyze functional sites of a protein or group of proteins. It identifies the conserved amino acid residues in an alignment and maps the information onto known 3D protein structures. This method exploits the fact that residues which are

ISSN 0973-2063

Bioinformation 2(9): 369-372 (2008) important to the structure or function of a protein tend to be conserved across species.

Advancement in protein engineering enables to design enzymes displaying activity and adaptation under optimized process conditions. The design of industrial enzymes is laborious, expensive and time consuming. Hence, prediction of desired properties using ET analysis is received among bio-catalysts scientists. Here, we describe the application of ET by computational mutagenesis towards the design of laccase with broad substrate specificity.

\section{Methodology: \\ Dataset}

Homologous sequences of laccase from Trametes versicolor with SWISS PROT accession number Q96UT7 were obtained from SWISSPROT database [7]. We used 31 protein sequences with identities more than $50 \%$ were selected using BLASTP [8] and aligned with ClustalW [9] using Gonnet protein weight matrix [10]. A rooted phylogenetic tree based on Neighbor-Joining algorithm was built from the multiple sequence alignment and visualized by PhyloDraw [11].

\section{Evolutionary trace analysis}

Multiple sequence alignment and phylogenetic tree were then used for ET analysis [6]. The sequences on different branches of the phylogram were assembled into different groups. The classes were generated by dividing the phylogram with evolutionary time cutoff lines. We then generated 4 different partition identity cutoffs (PICs), P1
Bioinformation, an open access forum (c) 2008 Biomedical Informatics Publishing Group 


\section{Bioinformation}

www.bioinformation.net to P4 based on the phylogram. In the phylogram, all the sequences originate from a common node in any given single partition generated by the partition cutoff line in the same group. Sequences within different groups, in a given partition were separately aligned, and the resultant aligned groups were compared to derive their consensus residues for specific partitions. Consensus residues from the multiple sequence alignment were classified as neutral, conserved and group-specific. Neutral residues are amino acids that are not conserved whereas conserved residues are the conserved in the multiple sequence alignment. Group-specific residues are amino acid residues that are conserved within the group, but they differ from one group to another. The trace residues were then mapped onto the known 3D structures of laccase (1KYA) [12] obtained from Protein Data Bank (PDB) [13] and the mapped structure visualized by Rasmol [14].

\section{Assignment of active site residues}

The crystal structure of laccase from PDB ID 1KYA complexed with 2, 5-xylidine (XYD) was visualized in Rasmol to identify the amino acid residues at the active site. We defined the ligand binding site in 1KYA as the amino acid residues within $5 \AA$ distance from the ligand, XYD. All the amino acids within the defined distance are considered as active site region in our analysis.

\section{Discussion:}

Laccase from Trametes versicolor was used as seed sequence in our analysis because it was reported as an important factor for successful bioremediation of phenolic wastewater by Trametes versicolor [15].

\section{Active site residues}

Ligand binding site analysis of 1KYA complexed with XYD ligand showed 10 amino acid residues within the distance defined as ligand binding site (Table 1 in supplementary material). These amino acid residues are considered as active site residues in our analysis. Among them, 206Asp has been reported to have a hydrophilic interaction with the ligand. Other residues involved in the hydrophobic protein-ligand interaction are 162Phe, 164Leu, 265Phe, 392Gly and 458His. Moreover, 458His forms hydrogen bonding with the ligand and coordinates the copper $\mathrm{C} 1$ atom that functions as the primary electron acceptor. On the other hand, 332Phe, 337Phe, 391 Pro and 455Ile are not showing any interaction with the ligand but they outline the cavity of the binding site.

\section{Phylogenetic tree and ET analysis}

We obtained 31 homologous sequences to paccase from Trametes versicolor with SWISS PROT accession number Q96UT7 from the SWISS PROT database. All the sequences analyzed originate from fungi with two different orders (Agaricales and Polyporales), except for Q12571 from Basisiomycetes PM1 which is yet to be classified. They vary in length from 517 to 533 residues

ISSN 0973-2063

Bioinformation 2(9): 369-372 (2008) with Q96UT7 having 520 residues should be noted. The homologous sequences were aligned and a rooted phylogenetic tree was generated as described in the methodology section. The phylogenetic tree is used in the ET algorithm based on the patterns of the members in the tree (Figure 1). The first node divides the phylogenetic tree into two major branches and we thus we defined it as our first cutoff partition (P1) in ET analysis. Members of the order of Agaricales (gilled mushrooms) are grouped in one branch in P1 except for Q6RYA5 from Flammulina velutipes. We generated another 3 cutoff partitions (P2, P3 and P4) in the ET analysis. Further analysis on $\mathrm{P} 4$ gave 5 groups as shown in Figure 1. Laccases from the order of Agaricales are grouped in G1 with cutoff partition P4. Q8WZG3 from Lentinula edodes is the only laccase from Agaricales outside G1 and it remains in cutoff partition P4 analysis as a member of G2. All members of the other groups are from the order of Polyporales which are basidiomycetes lacking soft gills, with all the Trametes and Pycnoporus species grouped in G4.

\section{Trace residues at the ligand binding site}

Consensus sequences derived from the entire cutoff partitions were aligned and compared with the amino acid sequence of Q96UT7 (Figure 2). We found 4 amino acids detected as conserved residues and no group specific residue was detected at the ligand binding site of 1KYA. The trace status of amino acid residues at the ligand binding site is given in Table 1 (supplementary material). The data shows that 392Gly was not a conserved residue until cutoff partition P4. Consistent with the findings in the structural data of 1KYA in which 206Asp and 458His are essentially contributed to the ligand binding site of laccase by the formation of hydrogen bonding with ligand [12]. We observed 206Asp and 458His within the defined ligand binding site cavity to be conserved.

We analyzed the amino acid sequence alignment of all laccases at the binding site to search for possible amino acid positions that could be manipulated by site-directed mutagenesis experiments to design laccase with broader substrate spectrum. All of the trace residues that are neutral at the binding site are non-polar amino acid (162Phe, 164Leu, 265Phe, 332Phe, 337Phe and 391Pro). In most cases, they show variation in alignment with other non-polar amino acids (Figure 3). We assumed that these are good sites for mutation. This is especially true with 162Phe that was aligned with much smaller nonpolar amino acids such as valine and leucine in the multiple sequence alignment (Figure 3 ). The alignment suggested that non-polar property is more important than aromatic property of phenylalanine at this position. Hence, we could suggest 162Phe 1as a good candidate for mutagenesis by changing with another non-polar amino acid yet small. This gives more space in the binding cavity for the entry of larger substrates. 


\section{www.bioinformation.net}

Hypothesis

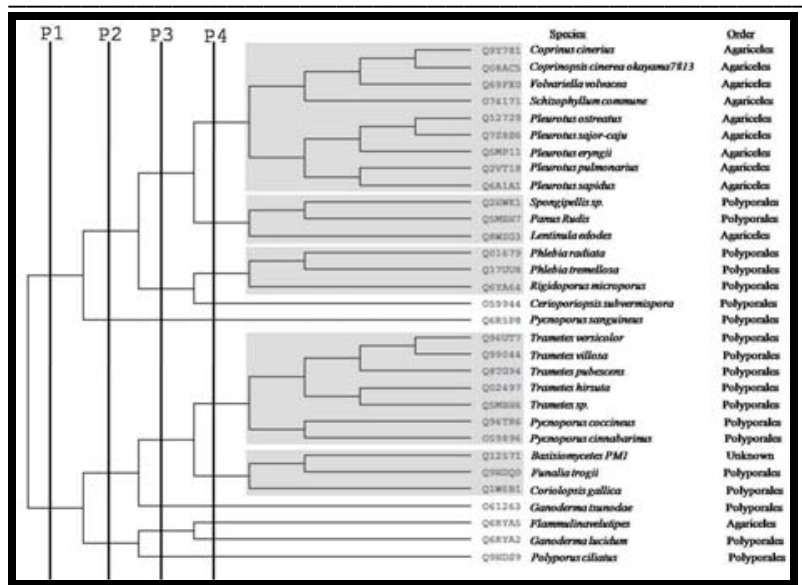

Figure 1: Evolutionary trace based dendrogram of selected laccases from SWISS PROT database. Partition cutoffs P1 to $\mathrm{P} 4$ are shown as vertical lines. Boxed are the group members of P4.

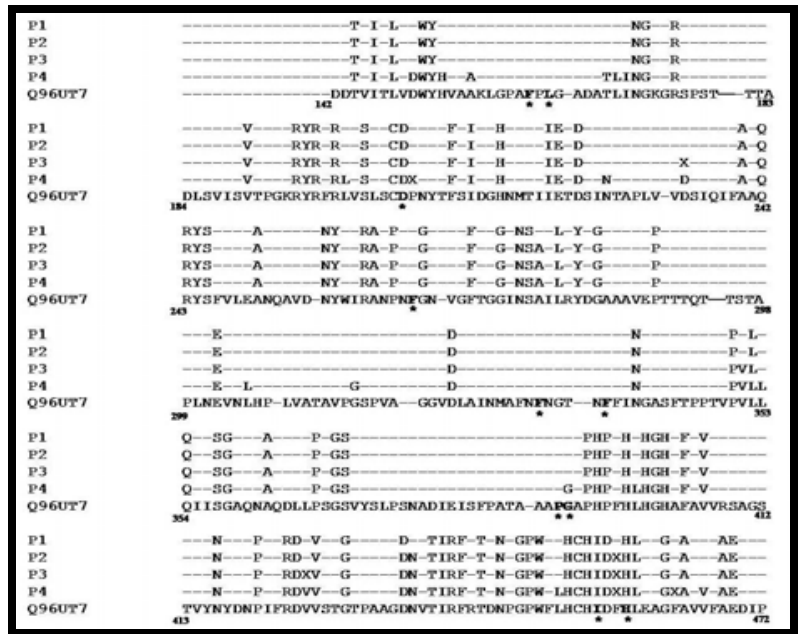

Figure 2: Consensus sequences from all partition aligned with amino acid sequence of Q96UT7. The numbering of amino acid sequence on Q96UT7 is according to the 1KYA. Asterisk amino acids were defined as ligand binding site amino acids.

\begin{tabular}{|c|c|c|c|c|c|c|}
\hline 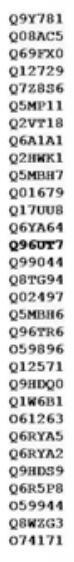 & 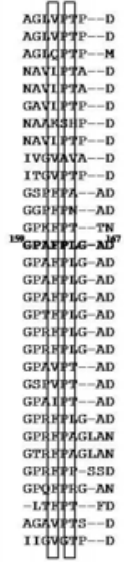 & 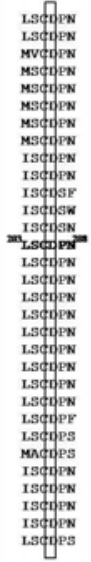 & 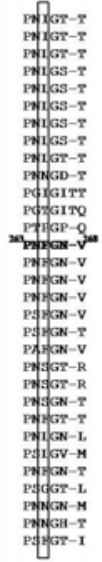 & 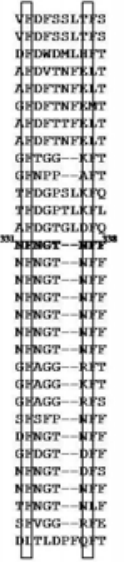 & 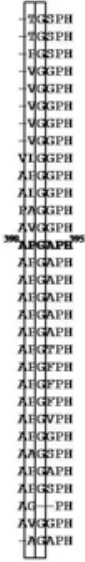 & 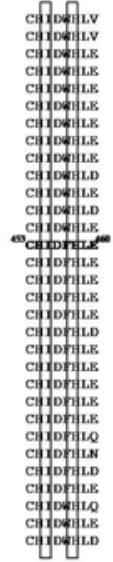 \\
\hline
\end{tabular}

Figure 3: Multiple sequence alignment of laccases at the ligand binding site of 1KYA.

ISSN 0973-2063

Bioinformation 2(9): 369-372 (2008) 


\section{Bioinformation}

\section{www.bioinformation.net}

\section{Hypothesis}

\section{Conclusion:}

We identified evolutionarily important amino acid residues at the ligand binding site of laccase using ET analysis. We also identified neutral residues at the binding site of laccase forming cavity in the binding site. These residues and in particular 162Phe is a target site for further structure-function experimental probing. Our findings provide a foundation to the design of laccase with a broader substrate spectrum for further expansion of laccase application in industry and bioremediation.

\section{References:}

[01] S. Riva, Trends Biotechnol., 24: 219 (2006) [PMID: 16574262]

[02] H. Claus, Micron, 35: 93 (2004) [PMID: 15036303]

[03] M. Mayer and R. C. Staples, Phytochemistry, 60: 551 (2002) [PMID: 12126701]

[04] P. Bajpai, et al., Biotechnol. Annu. Rev., 12: 349 (2006) [PMID: 17045199]
[05] T. Fukuda, et al., Biochem. Biophys. Res. Commun., 284: 704 (2001) [PMID: 11396959]

[06] O. Lichtarge, et al., J. Mol. Biol., 257: 342 (1996) [PMID: 8609628]

[07] http://www.expasy.org/

[08] http://www.ncbi.nlm.nih.gov/blast/

[09] J. D. Thompson, et al., Nucleic Acids Res., 22: 4673 (1994) [PMID: 7984417]

[10] G. H. Gonnet, et al., Science, 256: 1443 (1992) [PMID: 1604319]

[11] J. H. Choi, et al., Bioinformatics, 16: 1056 (2000) [PMID: 11159323]

[12] T. Bertrand, et al., Biochemistry, 41: 7325 (2002) [PMID: 12044164]

[13] http://www.rcsb.org/pdb/

[14] R. A. Sayle and E. J. Milner-White, Trends Biochem. Sci., 20: 374 (1995) [PMID: 7482707]

[15] D. Ryan, et al., Bioresour. Technol., 98: 579 (2007) [PMID: 16545562]

Edited by P. Kangueane

Citation: Mohamad et al., Bioinformation 2(9): 369-372 (2008)

License statement: This is an open-access article, which permits unrestricted use, distribution, and reproduction in any medium, for non-commercial purposes, provided the original author and source are credited.

\section{Supplementary material}

\begin{tabular}{lll}
\hline Residue no. & ${ }^{\text {a Amino acid }}$ & Trace status \\
\hline 162 & Phe & Neutral \\
164 & Leu & Neutral \\
206 & Asp & Conserved \\
265 & Phe & Neutral \\
332 & Phe & Neutral \\
337 & Phe & Neutral \\
391 & Pro & Neutral \\
392 & Gly & Conserved \\
455 & Ile & Conserved \\
458 & His & Conserved \\
\hline
\end{tabular}

Table 1: Amino acid residues at the ligand binding of $1 \mathrm{KYA}$ with their trace status. ${ }^{\mathrm{a}}$ The residue numbering is according to $1 \mathrm{KYA}$. 